\title{
Dielectric function for free electron gas: comparison between Drude and Lindhard models
}

Função dielétrica de um gás de elétrons: comparação entre os modelos de Drude e de Lindhard

\author{
A. V. Andrade-Neto*1 \\ ${ }^{1}$ Departamento de Física, Universidade Estadual de Feira de Santana, Feira de Santana, BA, Brazil
}

Received on September 14, 2016. Revised on October 31, 2016. Accepted on November 5, 2016.

\begin{abstract}
The interaction between light and metals or heavily doped semiconductors is largely determined by their free conduction electrons. The frequency and wave vector dependent complex dielectric function is an essential ingredient of the description of its optical and transport properties. The aim of this paper is to give a didactic introduction how the conduction electrons in solids responds to an external time dependent electric field and to make a comparison between Drude and Lindhard dielectric function models for the electron gas. In within framework of Lindhard model we derived an expression for dielectric function that is similar to the familiar Drudes's formula. In particular, the differences and similarities between the complex conductivity obtained from the two models are analyzed.
\end{abstract}

Keywords: dielectric function, Drude model, Lindhard model.

As propriedades físicas de metais e semicondutores dopados são em grande parte determinadas pelos seus elétrons de condução. A função dielétrica é uma quantidade física fundamental no estudo das propriedades óticas e de transporte desses materiais. Neste artigo discutimos de forma introdutória como os elétrons de condução em um sólido respondem à presença de um campo elétrico externo bem como realizamos uma comparação entre os modelos de Drude e de Lindhard no cálculo da função dielétrica de um gás de elétrons em um sólido. Em particular, as expressões para a condutividade complexa obtida dos dois modelos são analisadas e comparadas.

Palavras-chave: função dielétrica, modelo de Drude, modelo de Lindhard.

\section{Introduction}

In some solids, namely metals and doped semiconductors, a few loosely bound valence electrons are assumed to be completely detached from their atoms and move around throughout the material forming an electron gas. In this model, we consider that the positive ions core form a uniform positive background. The optical response of these materials can be described by means of a frequency and wave vector dependent complex dielectric function $\epsilon(\vec{q}, \omega)$ of the electron gas, which is an essential ingredient of the description of the transport and optical properties of solids [1]. Here, we will first review some of the basic aspects of response function, with

*Endereço de correspondência: aneto@uefs.br particular emphasis on relationship between electric susceptibility and dielectric function.

In a material media the relationship between the electric displacement $\vec{D}(\vec{r}, t)$ and the electric field $\vec{E}(\vec{r}, t)$ is given by (in cgs system)

$$
\vec{D}(\vec{r}, t)=\vec{E}(\vec{r}, t)+4 \pi \vec{P}(\vec{r}, t)
$$

where $\vec{P}(\vec{r}, t)$ is the macroscopic polarization (dipole moment per unit volume) that represents the response of the medium to an external electric field. This response for linear media that exhibit temporal and spatial dispersion, i.e., the response at position $\vec{r}$ and time $t$ to an electric field $\vec{E}\left(\vec{r}^{\prime}, t^{\prime}\right)$ at position $\vec{r}$ and time $t^{\prime}$ is given by 


$$
P_{i}(\vec{r}, t)=\sum_{j} \int d^{3} r^{\prime} \int \chi_{i j}\left(\vec{r}, \vec{r}^{\prime}, t, t^{\prime}\right) E_{j}\left(\vec{r}^{\prime}, t^{\prime}\right) d t^{\prime}
$$

where $i$ and $j$ refers to the components of the polarization $\vec{P}$ and electric field $\vec{E}$ and $\chi_{i j}$ are the components of the second rank tensor called electric susceptibility. For homogeneous medium the response function depends only on $\vec{r}-\vec{r}$ and Eq. (2) can be written as

$$
P_{i}(\vec{r}, t)=\sum_{j} \int d^{3} r^{\prime} \int \chi_{i j}\left(\vec{r}-\vec{r}, t-t^{\prime}\right) E_{j}\left(\vec{r}^{\prime}, t^{\prime}\right) d t^{\prime}
$$

The above equations simplify significantly by taking the Fourier transform. Considering an unitary volume of the sample, an electromagnetic field can be written as a superposition of monochromatic plane waves which components are given by

$$
E_{i}(\vec{r}, t)=\sum_{\vec{q}} \int_{-\infty}^{\infty} \frac{d \omega}{2 \pi} E_{i}(\vec{q}, \omega) \exp [\imath(\vec{q} \cdot \vec{r}-\omega t)]
$$

where $\vec{q}$ and $\omega$ are the wavevector and the angular frequency, respectively and $\vec{E}(\vec{q}, \omega)$ is the Fourier transform of the electric field $\vec{E}(\vec{r}, t)$ that is given by

$$
\vec{E}(\vec{q}, \omega)=\int d^{3} r \int_{-\infty}^{\infty} \vec{E}(\vec{r}, t) \exp [-\imath(\vec{q} \cdot \vec{r}-\omega t)] d t
$$

Similar equations apply to the electric displacement $\vec{D}(\vec{r}, t)$ and macroscopic polarization $\vec{P}(\vec{r}, t)$. Using this results the Eq. (1) becomes

$$
D_{i}(\vec{q}, \omega)=E_{i}(\vec{q}, \omega)+4 \pi P_{i}(\vec{q}, \omega) .
$$

Similarly, we can turn the convolution in Eq. (3) into multiplication, as we show in Appendix A

$$
P_{i}(\vec{q}, \omega)=\sum_{j} \chi_{i j}(\vec{q}, \omega) E_{j}(\vec{q}, \omega)
$$

Using Eq. (7) into Eq. (6) we have

$$
\epsilon_{i j}(\vec{q}, \omega)=1+4 \pi \chi_{i j}(\vec{q}, \omega),
$$

where $\epsilon_{i j}$ are the components of the dielectric tensor.

For an isotropic and homogeneous medium the susceptibility and dielectric tensors become scalars.
With these assumptions the Eqs. (7) and (8) can be written as

$$
\begin{gathered}
\vec{P}(\vec{q}, \omega)=\chi(\vec{q}, \omega) \vec{E}(\vec{q}, \omega), \\
\epsilon(\vec{q}, \omega)=1+4 \pi \chi(\vec{q}, \omega),
\end{gathered}
$$

Only isotropic and homogeneous medium will be considered in this work, and then $\epsilon(\vec{q}, \omega)$ is a scalar complex function, i.e., $\epsilon(\vec{q}, \omega)=\epsilon^{\prime}(\vec{q}, \omega)+\imath \epsilon^{\prime \prime}(\vec{q}, \omega)$.

Let us see the physical meaning of the real and imaginary parts of the dielectric function. The real part determines the amount of polarization when the material is subjected to an electric field while the imaginary part determines the amount of absorption inside the medium. Also, the real and imaginary parts of the dielectric function are related by the Kramers-Kronig relations which are typical of physical systems which obey causality and linearity conditions 2 .

There have been many approximations to the dielectric function. Here we present two dielectric models. In Section 2 we present the Drude approach which is the simplest model of the frequencydependent dielectric function of metals and semiconductors. Advances in quantum mechanics led to a more rigorous treatment for an electron gas system and in Section 3 we present the Lindhard model. The aim of this paper is to make a comparison about characteristics between Drude and Lindhard dielectric function expressions.

\section{Dielectric function from the Drude model}

Three years after the discovery of the electron by J. J. Thomson in 1897, Paul Drude developed a model which provides an effective description of the transport and optical properties of solids [3]. The Drude model is applied mainly to metals, but it is equally applicable to heavily doped semiconductors. It is assumed that each atom in a metal loses its valence electrons and becomes a positively charged ion. In a solid the discrete levels of the free atoms are broadening into bands. In metals the highest band containing electrons is called conduction band which is filled with electrons that originates from the atom's outermost orbitals [4]. In the Drude model, these electrons do not interact with each other and are scattered randomly by ionic cores, i.e., the con- 
duction electrons are considered as independent and quasi-free particles.

We want to know how the conduction electrons respond to an external probe like a time dependent electric field. The motion of these electrons is damped via collisions that occur with a characteristic collision frequency $\gamma=1 / \tau$ where $\tau$ is an average relaxation time, which for a typical metal is the order of $10^{-14} \mathrm{~s}$.

The equation of motion can be written as

$$
m^{*} \ddot{\vec{r}}+m^{*} \gamma \dot{\vec{r}}=-e \vec{E}(t) .
$$

where $r$ is the ensemble average of the displacement of the conduction electrons, $m^{*}$ is the electron effective mass that incorporate the band structure of the material and $-e$ is the electronic charge. The second term proportional to the drift velocity represents the frictional force and the dots denote differentiation with respect to time. Drude model can be extended by adding a restoring force, which constitutes the Drude-Lorentz model, (see, for example, the reference [5]).

In the long wavelength limit $q \rightarrow 0$ we can write the electric field as

$$
\vec{E}(t)=\int_{-\infty}^{\infty} \frac{d \omega}{2 \pi} \vec{E}(\omega) \exp (-\imath \omega t),
$$

with similar equation for $\vec{r}(t)$. Inserting the above Fourier representation for $\vec{E}(t)$ and $\vec{r}(t)$ into Eq. (11) we obtain

$$
\vec{r}(\omega)=\frac{e \vec{E}(\omega)}{m^{*}\left(\omega^{2}+\imath \gamma \omega\right)} .
$$

The displaced electrons, due to the electric field, contribute to the macroscopic polarization $\vec{P}=$ $-n e \vec{r}$, where $n$ is the density of charge carriers. Taking the Fourier transform we get using Eq.(13)

$$
\vec{P}(\omega)=-\frac{n e^{2}}{m^{*}\left(\omega^{2}+\imath \gamma \omega\right)} \vec{E}(\omega) .
$$

We get from Eq. 9 (9)

$$
\chi(\omega)=-\frac{n e^{2}}{m^{*}\left(\omega^{2}+\imath \gamma \omega\right)}
$$

and from Eq. 10.

$$
\epsilon_{D}(\omega)=1-\frac{\omega_{p l}^{2}}{\omega^{2}+\imath \gamma_{D} \omega}
$$

where the subscript $\mathrm{D}$ refers to Drude and

$$
\omega_{p l}^{2}=\frac{4 \pi n e^{2}}{m^{*}}
$$

is the plasma frequency of the free electron gas which behaves as a critical value for propagation of the radiation through a material. At frequencies $\omega$ above $\omega_{p l}$ the absorption is small and the radiation can propagate. At frequencies $\omega$ below $\omega_{p l}$ there will be absorption and the radiation will drop off exponentially through a material. We can express $\omega_{p l}$ in terms of an energy $\hbar \omega_{p l}$, where $\hbar$ is the Planck constant. The energy quanta of these collective oscillations of the electron plasma are called plasmons, which, for noble metals is of the order of $10 \mathrm{eV}$.

The Drude model considers only the conduction electrons. To account the net contribution from the positive ion cores we can introduced the parameter $\epsilon_{\infty}$. Then it is possible to rewrite the Eq. (16) as

$$
\epsilon_{D}(\omega)=\epsilon_{\infty}-\frac{\omega_{p l}^{2}}{\omega^{2}+\imath \gamma_{D} \omega}
$$

The dielectric function in Eq. (18) can be decomposed into real $\epsilon^{\prime}$ and imaginary part $\epsilon^{\prime \prime}$

$$
\begin{gathered}
\epsilon_{D}^{\prime}(\omega)=\epsilon_{\infty}-\frac{\omega_{p l}^{2}}{\omega^{2}+\gamma_{D}^{2}} \\
\epsilon_{D}^{\prime \prime}(\omega)=\frac{\omega_{p l}^{2} \gamma_{D}}{\omega\left(\omega^{2}+\gamma_{D}^{2}\right)}
\end{gathered}
$$

The imaginary part of the dielectric function diverges when $\omega \rightarrow 0$. This is an unphysical behaviour.

Despite its simplicity, the Drude model parameters has been utilized to fit experimental results [6], in particular in terahertz time-domain spectroscopy (THz-TDS) [7], which is a contactless technique in which the properties of solids are probed with short pulses of terahertz radiation. However, a more realistic description of the electron gas system requires a quantum mechanical model. In the next Section we present the Lindhard model for dielectric function.

\section{Dielectric function from the Lindhard model}

A more rigorous quantum mechanics treatment of many-electron systems was carried out by Lindhard in 1954, which used the Random-Phase Approximation (RPA). He derived a formula for the dielectric function that described both the collective behavior 
at small $q$ values and the single-particle excitations at large $q$ values.

A deduction to Lindhard dielectric function can be found in Refs $[1,8,9$. Here we quote the final expression which is given by

$$
\begin{aligned}
& \epsilon_{L}(\vec{q}, \omega)=\epsilon_{\infty} \\
& -V(q) \sum_{\vec{k}} \frac{f(\vec{k}+\vec{q})-f(\vec{k})}{E(\vec{k}+\vec{q})-E(\vec{k})-\hbar(\omega+\imath s)},
\end{aligned}
$$

where $V(q)=4 \pi e^{2} / q^{2}$ is the Fourier transform of the Coulomb potential, $f(\vec{k})$ is the Fermi-Dirac distribution function, $E(\vec{k})$ is the electron energy, $s$ is a positive infinitesimal constant and the subscript L refers to Lindhard. The Eq. (21) is a general expression that includes spatial dispersion ( $q$ dependence) and temporal dispersion ( $\omega$ dependence). The Eq.(21) can be used also for nonequilibrium distribution functions 10 .

Analytical closed expressions for the Lindhard dielectric function at finite temperature are hardly to obtain [11. General analytic expression can be evaluated analytically for the two limiting cases. For $T=0[9]$ where the Fermi-Dirac distribution is the unit-step function and high temperature limits 11 where the distribution function is aproximated as Maxwell-Boltzmann distribution.

In order to obtain a similar Drude expression we will replace in the Eq. 21) the infinitesimal constant $s$ by a finite parameter $\gamma_{L}$ which is a phenomenological decay constant. Then, Eq. 21) yields

$$
\begin{aligned}
& \epsilon_{L}(\vec{q}, \omega)=\epsilon_{\infty} \\
& -V(q) \sum_{\vec{k}} \frac{f(\vec{k}+\vec{q})-f(\vec{k})}{E(\vec{k}+\vec{q})-E(\vec{k})-\hbar\left(\omega+\imath \gamma_{L}\right)} .
\end{aligned}
$$

The equation above can be rewritten as

$$
\begin{aligned}
& \epsilon_{L}(\vec{q}, \omega)=\epsilon_{\infty} \\
& -2 V(q) \sum_{\vec{k}} \frac{f(\vec{k})[E(\vec{k}+\vec{q})-E(\vec{k})]}{\hbar^{2}\left(\omega+\imath \gamma_{L}\right)^{2}-[E(\vec{k}+\vec{q})-E(\vec{k})]^{2}} .
\end{aligned}
$$

In the long wavelength limit $q \rightarrow 0$ the quantity $E(\vec{k}+\vec{q})-E(\vec{k})$ can be neglected in the denominator of (23), that can be written

$$
\epsilon_{L}(\vec{q}, \omega)=\epsilon_{\infty}-2 V(q) \sum_{\vec{k}} \frac{f(\vec{k})[E(\vec{k}+\vec{q})-E(\vec{k})]}{\hbar^{2}\left(\omega+\imath \gamma_{L}\right)^{2}}
$$

Using parabolic band approximation and considering the high temperature limit for the distribution function, the Eq. (24) becomes (we present the details in Appendix B)

$$
\epsilon_{L}(\omega)=\epsilon_{\infty}-\frac{\omega_{p l}^{2}}{\left(\omega+\imath \gamma_{L}\right)^{2}}
$$

where $\omega_{p l}$ is the plasma frequency given by Eq. (17). Eq. (25) is the central equation of this paper and can be compared with Eq.(18). If we compare the Drude formula, Eq. (18), with the Lindhard formula, Eq. (25), one sees that an advantage of the second expression is that it does not diverge when $\omega \rightarrow 0$. In the case of negligible damping the Eqs. (18) and (25) coincide.

The real and imaginary parts of this complex dielectric function are given by

$$
\begin{gathered}
\epsilon_{L}^{\prime}(\omega)=\epsilon_{\infty}-\frac{\omega_{p l}^{2}\left(\omega^{2}-\gamma_{L}^{2}\right)}{\left(\omega^{2}+\gamma_{L}^{2}\right)^{2}}, \\
\epsilon_{L}^{\prime \prime}(\omega)=\frac{2 \omega_{p l}^{2} \gamma_{L} \omega}{\left(\omega^{2}+\gamma_{L}^{2}\right)^{2}} .
\end{gathered}
$$

We can see from the above equations that the real part $\epsilon_{L}^{\prime}(-\omega)=\epsilon_{L}^{\prime}(\omega)$ is an even function and the imaginary part $\epsilon_{L}^{\prime \prime}(-\omega)=-\epsilon_{L}^{\prime \prime}(\omega)$ is an odd function, a well-known property of dielectric function [12. Other mathematical properties will be explored in Appendix C.

The interesting feature of this result is the fact that the imaginary part does no diverge when $\omega \rightarrow 0$, unlike what happens in the Drude model.

The frequency dependencies of the real and imaginary parts of the dielectric function are shown in Figures 1 and 2, respectively. Black lines results from Drude model, Eqs. (19) and 20), while the red lines was obtained using the Lindhard model, Eqs. (26) and (27). The parameters used were $n=$ $7.8 \times 10^{15} \mathrm{~cm}^{-3}$ (the same value of the reference 13$]$ ), $\gamma_{D}=0.96 \mathrm{THz} \gamma_{L}=0.48 \mathrm{THz}, \epsilon_{\infty}=10.89$ and $m^{*}=0.067 m_{e}$ (parameters of Gallium Arsenide).

From Figures 1 and 2 we can see that for large frequency values there is little difference between Drude and Lindhard predictions for the dielectric function. On the other hand, there is a great difference in the predictions of the two models in the 


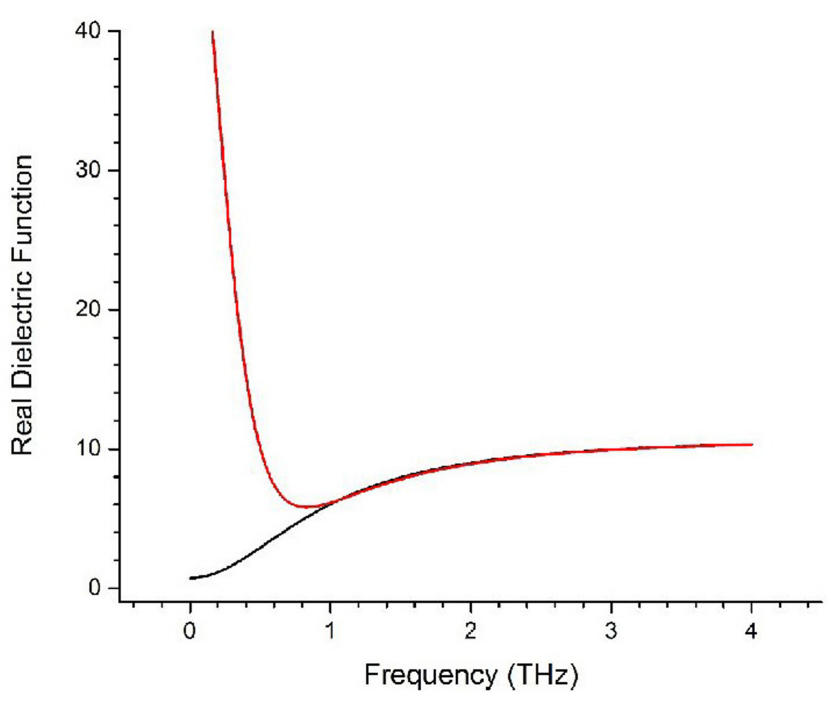

Figure 1: Real part of the dielectric function of the Drude (black line) and Lindhard (red line) models as a function of frequency for $n=7.8 \times 10^{15} \mathrm{~cm}^{-3}$ (the same value of the reference [13]), $\gamma_{D}=0.96 \mathrm{THz} \gamma_{L}=0.48 \mathrm{THz}$, $\epsilon_{\infty}=10.89$ and $m^{*}=0.067 m_{e}$. The black line is obtained from Eq.(19) and the red line is obtained from Eq.(26).

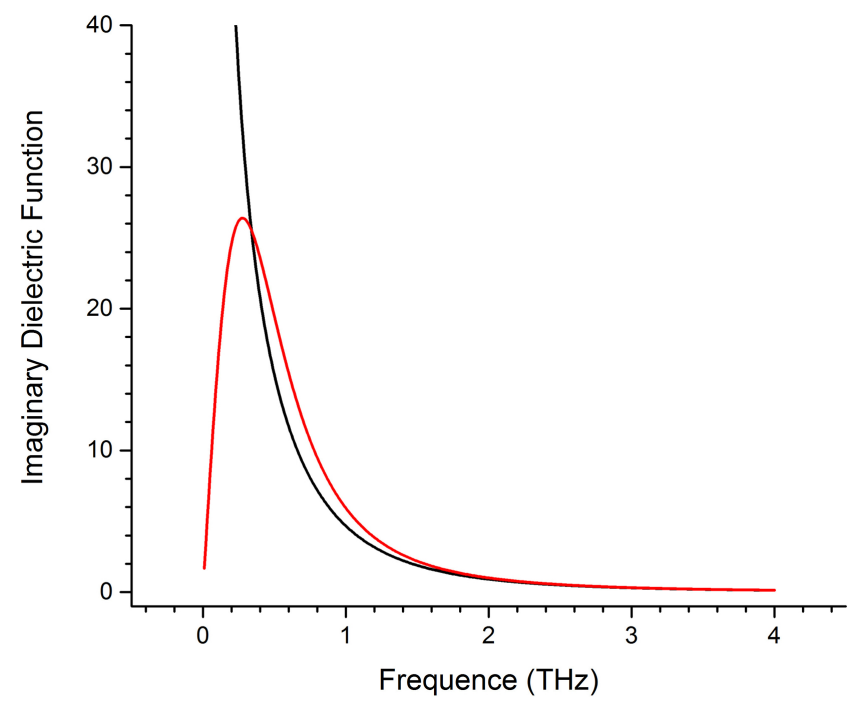

Figure 2: Imaginary part of the dielectric function as a function of frequency. The black line is obtained from Drude, Eq.(20), and the red line is obtained from Lindhard model, Eq. (27). The parameter values are the same as in Figure 1.

low frequency region, which can be confronted with experiments in terahertz time-domain spectroscopy (THz-TDS)

\section{Conductivity}

It is often convenient to express the response of the electron gas in terms of the conductivity $\sigma(\omega)$, which is directly related to the dielectric function $\epsilon(\omega)$. From Maxwell's equations, we can obtain the follow relationship between $\epsilon(\omega)$ and $\sigma(\omega)[3]$ (in cgs system)

$$
\epsilon(\omega)=\epsilon_{\infty}+\frac{4 \pi \imath}{\omega} \sigma(\omega)
$$

that can be rewritten as

$$
\sigma(\omega)=-\frac{\imath \omega}{4 \pi}\left[\epsilon(\omega)-\epsilon_{\infty}\right] .
$$

Measurements of the complex conductivity of moderately doped semiconductors, using terahertz time-domain spectroscopy, has been widely reported [6, 13, 14].

Using the expressions for the dielectric function given by the Drude and Lindhard models we can write the conductivity as

\subsection{Drude Model}

$$
\begin{aligned}
& \sigma_{D}^{\prime}(\omega)=\frac{\sigma_{o D} \gamma_{D}^{2}}{\omega^{2}+\gamma_{D}^{2}} \\
& \sigma_{D}^{\prime \prime}(\omega)=\frac{\sigma_{o D} \gamma_{D} \omega}{\omega^{2}+\gamma_{D}^{2}}
\end{aligned}
$$

where

$$
\sigma_{o D}=\frac{n e^{2}}{m^{*} \gamma_{D}}
$$

\subsection{Lindhard Model}

$$
\begin{gathered}
\sigma_{L}^{\prime}(\omega)=\frac{4 \sigma_{o L} \gamma_{L}^{2} \omega^{2}}{\left(\omega^{2}+\gamma_{L}^{2}\right)^{2}} \\
\sigma_{L}^{\prime \prime}(\omega)=\frac{2 \sigma_{o L} \gamma_{L} \omega\left(\omega^{2}-\gamma_{L}^{2}\right)}{\left(\omega^{2}+\gamma_{L}^{2}\right)^{2}}
\end{gathered}
$$

where

$$
\sigma_{o L}=\frac{n e^{2}}{2 m^{*} \gamma_{L}}
$$

\section{Results and remarks}

Figures 3 and 4 show the frequency dependence of the real and the imaginary parts of the complex conductivity, respectively, calculated from Drude (black line) and Lindhard models (red line). In Figure 3 is shown the real part of the conductivity calculated from Eq. (30) (black line) and Eq. (33) (red line). Figure 4 shows the imaginary part calculated from 


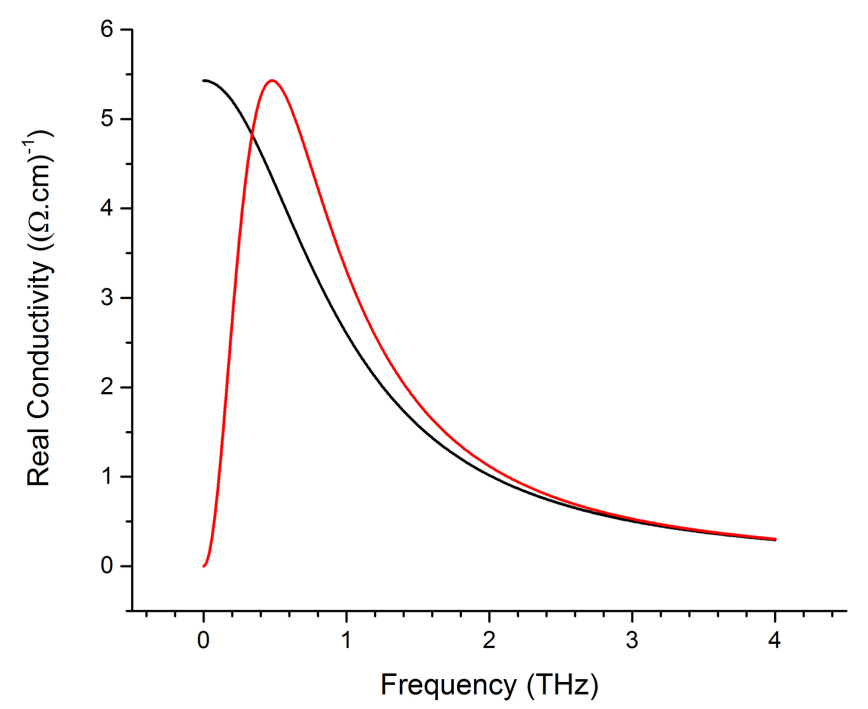

Figure 3: Real part of the complex conductivity as a function of frequency calculated from Drude and Lindhard models. Black line: Real part calculated from Eq. (30). Red line: Real part calculated from Eq.(33). The parameter values are the same as in Figures 1 and 2.

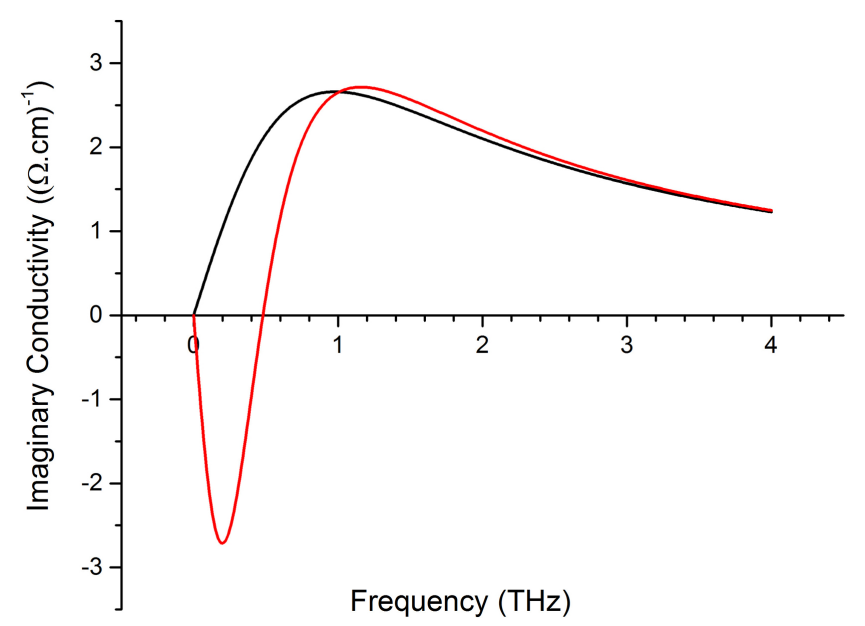

Figure 4: Imaginary part of the complex conductivity as a function of frequency calculated from Drude and Lindhard models. Black line: Imaginary part calculated from Eq.(31). Red line: Imaginary part calculated from Eq.(34). The parameter values are the same as in Figures 1 and 2 .

Eq.(31) (black line) and Eq.(34) (red line). The parameter values are the same as in Figures 1 and 2.

From the above results, we can to highlight the following characteristics:

1 - In the Drude model the real part of the conductivity has a finite value for $\omega=0$ while in the Lindhard model this value vanish for $\omega=0$, i.e.,

$$
\sigma_{D}^{\prime}(\omega=0)=\sigma_{o D}
$$

and

$$
\sigma_{L}^{\prime}(\omega=0)=0
$$

2 - In the Drude model the imaginary part of the conductivity is always positive, while in the Lindhard model the imaginary part of the conductivity is negative for values in the range $0<\omega<\gamma_{L}$. The negative imaginary conductivities are reported in semiconductors nanomaterials 15.

3 - In the Lindhard model, the real part of the conductivity exhibits a maximum at $\omega=\gamma_{L}$ before it drops to zero for $\omega \rightarrow 0$, while the imaginary part is null at $\omega=\gamma_{L}$, i.e., $\sigma_{L}^{\prime \prime}\left(\omega=\gamma_{L}\right)=0$.

4 - Our expressions, derived from the Lindhard model, provide significantly better agreement with experimental results for complex conductivity than the Drude model. See, for example, Figure 3 from Reference [6] where we can observe that the shape of the experimental points, in the low frequency region, is better comparable to that obtained from Lindhard model.

\section{Conclusion}

The response of the material medium to the electromagnetic fields can be described in terms of the frequency and wavevector dependent complex dielectric function and conductivity.

In this work in within framework of Lindhard model we have presented an expression for dielectric function, Eq. (25), that is similar to the familiar Drudes's formula, Eq.(18). For large $\omega$ there is little difference between Drude and Lindhard predictions for the dielectric function expressions. However, for $\omega \rightarrow 0$ the Drude model predict that the imaginary part tends to infinity. In contrast, the expression derived from the Lindhard model does not diverge in this limit.

In summary, in this manuscript we presented an equation for the dielectric function which, on the one hand, as simple as Drude's equation but, on the other hand, is finite for all values of $\omega$, including zero. Starting from this equation, we derive expressions for the complex conductivity from the Lindhard model that provide significantly better agreement with experimental results for conductivity than the Drude model. 


\section{Appendix A}

The components of $P_{i}(\vec{r}, t)$ and $E_{i}(\vec{r}, t)$ can be written as

$$
P_{i}(\vec{r}, t)=\sum_{\vec{q}} \int_{-\infty}^{\infty} \frac{d \omega}{2 \pi} P_{i}(\vec{q}, \omega) \exp [\imath(\vec{q} \cdot \vec{r}-\omega t)],
$$

$$
E_{i}(\vec{r}, t)=\sum_{\vec{q}} \int_{-\infty}^{\infty} \frac{d \omega}{2 \pi} E_{i}(\vec{q}, \omega) \exp [\imath(\vec{q} \cdot \vec{r}-\omega t)],
$$

Using A.1 and A.2 in Eq. (3) we get

$$
\begin{aligned}
& \sum_{\vec{q}} \int_{-\infty}^{\infty} P_{i}(\vec{q}, \omega) \exp [\imath(\vec{q} \cdot \vec{r}-\omega t)] d \omega \\
& =\sum_{j} \sum_{\vec{q}} \int d^{3} r^{\prime} \int_{-\infty}^{\infty} d t^{\prime} \chi_{i j}\left(\vec{r}-\vec{r}^{\prime}, t-t^{\prime}\right) \\
& \times \int_{-\infty}^{\infty} d \omega E_{j}(\vec{q}, \omega) \exp \left[\imath\left(\vec{q} \cdot \vec{r}^{\prime}-\omega t^{\prime}\right)\right]
\end{aligned}
$$

Making the change of variables $\vec{r}-\vec{r}^{\prime}=\vec{r}^{\prime \prime}$ and $t-t^{\prime}=t^{\prime \prime}$ we obtain

$$
\begin{aligned}
& \sum_{\vec{q}} \int d \omega \exp [\imath(\vec{q} \cdot \vec{r}-\omega t)]\left[P_{i}(\vec{q}, \omega)\right. \\
& \left.-\sum_{j} \chi_{i j}(\vec{q}, \omega) E_{j}(\vec{q}, \omega)\right]=0
\end{aligned}
$$

where

$$
\begin{aligned}
& \chi_{i j}(\vec{q}, \omega)=\int d^{3} r^{\prime \prime} \int d t^{\prime \prime} \chi_{i j}\left(\vec{r}^{\prime \prime}, t^{\prime \prime}\right) \\
& \times \exp \left[-\imath\left(\vec{q} \cdot \vec{r}^{\prime \prime}-\omega t^{\prime \prime}\right)\right] .
\end{aligned}
$$

From Eq.(A.4) we obtain

$$
P_{i}(\vec{q}, \omega)=\sum_{j} \chi_{i j}(\vec{q}, \omega) E_{j}(\vec{q}, \omega)
$$

which is Eq. (7).

\section{Appendix B}

Eq. (25) is derived as follows. Transforming the sum over $k$ in an integral by the known rule $\sum_{k} \rightarrow$ $\left(2 / 8 \pi^{3}\right) \int d^{3} k$ the Eq. B.5) becomes

$$
\begin{aligned}
& \epsilon_{L}(\vec{q}, \omega)=\epsilon_{\infty}-\frac{V(q)}{2 \pi^{3} \hbar^{2}\left(\omega+\imath \gamma_{L}\right)^{2}} \\
& \times \int f(\vec{k})[E(\vec{k}+\vec{q})-E(\vec{k})] d^{3} k .
\end{aligned}
$$

where $V(q)=4 \pi e^{2} / q^{2}$. Using parabolic band approximation, i.e.,

$$
E(\vec{k}+\vec{q})-E(\vec{k})=\frac{\hbar^{2}}{2 m}\left(2 \vec{k} \cdot \vec{q}+\vec{q}^{2}\right),
$$

and spherical coordinates $d^{3} k=2 \pi k^{2} d k \sin \theta d \theta$, where $\theta$ is the angle between $\vec{k}$ and $\vec{q}$, we obtain

$$
\begin{aligned}
& \epsilon_{L}(\vec{q}, \omega)=\epsilon_{\infty}-\frac{V(q) q^{2}}{2 m^{*} \pi^{2}\left(\omega+\imath \gamma_{L}\right)^{2}} \\
& \times \int f(\vec{k}) k^{2} d k \sin \theta d \theta .
\end{aligned}
$$

Considering the high temperature limit the distribution function can be written as [8]

$$
f(k)=\frac{4 \pi^{3} n \hbar^{3}}{\left(2 \pi m^{*} k_{B} T\right)^{3 / 2}} \exp \left(\frac{-\hbar^{2} k^{2}}{2 m^{*} k_{B} T}\right)
$$

where $n$ is the density of charge carriers, $k_{B}$ is the Boltzmann constant and $T$ is the absolute temperature.

Using (B.4) in (B.3) we obtain after integration in angular part

$$
\begin{aligned}
& \epsilon_{L}(\vec{q}, \omega)=\epsilon_{\infty}-\frac{16 \pi^{3} n e^{2} \hbar^{3}}{\pi m\left(2 \pi m^{*} k_{B} T\right)^{3 / 2}} \frac{1}{\left(\omega+\imath \gamma_{L}\right)^{2}} \\
& \times \int_{0}^{\infty} \exp \left(-\alpha k^{2}\right) k^{2} d k .
\end{aligned}
$$

where $\alpha=\hbar^{2} /\left(2 m^{*} k_{B} T\right)$. Since

$$
\int_{0}^{\infty} \exp \left(-\alpha k^{2}\right) k^{2} d k=\frac{\pi^{1 / 2}}{4 \alpha^{3 / 2}}
$$

we obtain

$$
\epsilon_{L}(\omega)=\epsilon_{\infty}-\frac{\omega_{p l}^{2}}{\left(\omega+\imath \gamma_{L}\right)^{2}}
$$

which is Eq.(25).

\section{Appendix $\mathrm{C}$}

Let us now show that the expressions found using Lindhard model, obeys certain important properties such as the sum rule for conductivity and KramersKronig relation. 


\section{Sum rule for the conductivity}

The sum rule for the real part of the conductivity is expressed as

$$
\int_{0}^{\infty} \operatorname{Re}[\sigma(\omega)] d \omega=\int_{0}^{\infty} \sigma^{\prime}(\omega) d \omega=\frac{\pi n e^{2}}{2 m}
$$

Making use of the Eq. (33), we have

$$
\int_{0}^{\infty} \sigma_{L}^{\prime}(\omega) d \omega=4 \sigma_{o L} \gamma_{L}^{2} \int_{0}^{\infty} \frac{\omega^{2}}{\left(\omega^{2}+\gamma_{L}^{2}\right)^{2}} d \omega
$$

Using

$$
\int_{0}^{\infty} \frac{x^{2}}{\left(x^{2}+a^{2}\right)^{2}} d x=\frac{\pi}{4 a}
$$

we obtain

$$
\int_{0}^{\infty} \sigma_{L}^{\prime}(\omega) d \omega=\frac{\pi n e^{2}}{2 m}
$$

\section{Kramers-Kronig relation}

From the Kramers-Kronig relations we obtain

$$
\int_{0}^{\infty} \omega \operatorname{Im}[\epsilon(\omega)] d \omega=\int_{0}^{\infty} \omega \epsilon^{\prime \prime}(\omega) d \omega=\frac{\pi}{2} \omega_{p l}^{2}
$$

Making use of the Eq. 20

$$
\int_{0}^{\infty} \omega \epsilon_{L}^{\prime \prime}(\omega) d \omega=2 \omega_{p l}^{2} \gamma_{L} \int_{0}^{\infty} \frac{\omega^{2}}{\left(\omega^{2}+\gamma_{L}^{2}\right)^{2}} d \omega
$$

Using the Eq. C.3 we obtain

$$
\int_{0}^{\infty} \omega \epsilon_{L}^{\prime \prime}(\omega) d \omega=\frac{\pi}{2} \omega_{p l}^{2}
$$

\section{Acknowledgments}

Financial support from CAPES is kindly acknowledged. The author thanks for hospitality of the NanoSpectroscopy Laboratory (LabNS) at Physics Department of the UFMG where this work was finished. The author also wishes to thank to Aroldo Ribeiro for graphics.

\section{References}

[1] Martin Dressel and George Grüner, Electrodynamics of Solids (Cambridge University Press, Cambridge, 2002).

[2] Márcio José Menon e Ricardo Paupitz Barbosa dos Santos, Revista Brasileira de Ensino de Física 20, 38 (1998).

[3] Neil W. Ashcroft and N. David Mermin, Solid State Physics (Saunders College Publishing, Fort Worth, 1976).

[4] Nilson S. de Andrade, A.V. Andrade-Neto, Thierry Lemaire e J.A. Cruz, Revista Brasileira de Ensino de Física 35, 1308 (2013).

[5] John R. Reitz, Frederick J. Milford e Robert W. Christy, Fundamentos da Teoria Eletromagnética (Editora Campus, Rio de Janeiro, 1994), 3rd ed., cap. 22.

[6] Martin Dressel and Marc Scheffler, Ann. Phys. 15, 535 (2006).

[7] Ronald Ulbricht, Euan Hendry, Jie Shan, Tony F. Heinz and Mischa Bonn, Rev. Mod. Phys. 83, 543 (2011).

[8] Hartmut Haug and Sthepan W. Koch, Quantum Theory of the Electronic Properties of Semiconductors (World Scientific, Singapore, 1994), 3rd ed., p. 97.

[9] G.D. Mahan, Many-Particle Physics (Plenum, New York, 1987)

[10] A.V. Andrade-Neto, A.R. Vasconcellos, R. Luzzi and V.N. Freire, Appl. Phys. Lett. 85, 4055 (2004).

[11] A.V. Andrade-Neto, arXiv:1412.5705 (2014).

[12] L.D. Landau, E.M. Lifshitz and L.P. Pitaevskii, Electrodynamics of Continuous Media (ButterworthHeinenann, Oxford, 1984), $2^{a}$ ed., v. 8, p. 266.

[13] N. Katzenellenbogen and D. Grischkowsky, Appl. Phys. Lett. 61, 840 (1992).

[14] Han-Kwang Nienhuys and Villy Sundstrom, Appl. Phys. Lett. 87, 012101 (2005).

[15] James Lloyd-Hughes and Tae-In Jeon, J. Infrared, Milim. Terahertz Waves 33, 871 (2012). 